\title{
Redundancy gain for semantic features
}

\author{
Anja Fiedler • Hannes Schröter • Rolf Ulrich
}

Published online: 19 December 2012

(C) Psychonomic Society, Inc. 2012

\begin{abstract}
In a go/no-go experiment, semantic redundancy gain was assessed for responses to single written words. Specifically, we asked participants to respond only to words whose meaning matched at least one semantic target featurethat is, the target category (e.g., animal), the target color (e.g., gray), or both. On redundant-target trials, the word (e.g., elephant) matched both semantic target features (i.e., gray and animal). On single-target trials, the word (e.g., beaver) matched one target feature (i.e., animal) and a nontarget feature (i.e., brown). We observed shorter reaction times in the redundant-target condition than in the faster single-target condition. Hence, the present study provides the first evidence that redundancy gain is not limited to responses to redundant proximal stimulus features but can also be observed for responses to semantic feature information.
\end{abstract}

Keywords Redundancy gain $\cdot$ Redundant targets $\cdot$ Semantic features $\cdot$ Mental representations

\section{Introduction}

It is quite unclear whether the same cognitive operations are involved when proximal stimuli located in the extrapersonal world are processed, as compared with processing internal representations that currently have no external referent (e.g., memories or concepts). For instance, it has been argued that searching for a visual target is guided by the same mechanism that underlies memory search (Gilford \& Juola, 1976). Yet, search slope ratios obtained in visual, as compared with memory, search often deviate from each other, suggesting that

A. Fiedler $\cdot$ H. Schröter $\cdot$ R. Ulrich

Department of Psychology, University of Tübingen,

Tübingen, Germany

\section{A. Fiedler $(\square)$}

Department of Cognition and Perception,

University of Tübingen, Schleichstraße 4,

72076 Tübingen, Germany

e-mail: anja.fiedler@uni-tuebingen.de search mechanisms differ between external stimuli and internal memory representations (for a review, see Van Zandt \& Townsend, 1993).

The redundant-target paradigm can be seen as one type of search task that has so far received rather little attention with regard to the question of whether proximal stimuli and mental representations are processed differently. In this paradigm, participants are presented with one or multiple targets that require the same response. Usually, reaction time (RT) for target-present responses decreases with an increasing number of targets. This redundancy gain has been observed in standard visual search paradigms (e.g., Töllner, Zehetleitner, Krummenacher, \& Müller, 2011), simple RT tasks (e.g., Fischer \& Miller, 2008), and go/no-go tasks (e.g., Mordkoff \& Danek, 2011). Traditionally, redundancy gain has been explained by statistical facilitation resulting from parallel and independent processing of redundant targets (race model; Raab, 1962). However, subsequent research has shown that the size of observed redundancy gains can violate the race model inequality (Miller, 1982), providing evidence against independent processing of redundant stimuli (e.g., Miller, 1982; Mordkoff \& Danek, 2011).

In previous redundancy gain experiments, the decision to make a response was based on proximal stimulus features. For instance, participants responded to the presentation of any visual (Fischer \& Miller, 2008), auditory (Schröter, Ulrich, $\&$ Miller, 2007), or multimodal stimuli (Diederich \& Colonius, 2004) or to specific target-defining features of stimuli like color and/or shape (Mordkoff \& Danek, 2011). In a series of simple RT studies, however, Schröter and colleagues (Schröter, Fiedler, Miller, \& Ulrich, 2011; Schröter, Frei, Ulrich, \& Miller, 2009; Schröter et al., 2007) demonstrated that redundant proximal targets are not sufficient to result in redundancy gain. Accordingly, no redundancy gain can be observed if redundant proximal targets fuse into a single percept (i.e., a single representation of distal stimuli). In addition, there is evidence that redundant proximal targets are also not necessary for the occurrence of a redundancy gain. Specifically, Fiedler, O'Sullivan, Schröter, Miller, and Ulrich (2011) showed that an illusionary redundant flash 
evoked by the sound-induced flash illusion (Shams, Kamitani, \& Shimojo, 2002) can result in a redundancy gain, even though there is no proximal redundancy. On the basis of this evidence, Schröter and colleagues concluded that the occurrence of redundancy gain does not depend on the number of proximal stimuli but, rather, on the number of percepts.

The aim of the present study is to go one step further. Specifically, we examine whether information processing is also facilitated if redundant targets are neither proximal stimuli nor percepts but, instead, have to be retrieved from semantic memory (i.e., redundant internal representations). Basically, semantic memory constitutes a subsystem of the declarative memory system, which contains conceptual knowledge about the world, including the meaning of words (e.g., Tulving, 1983). Importantly, each concept within semantic memory is not stored in isolation but is interconnected with related concepts and corresponding knowledge. For example, if we are familiar with the concept elephant, this concept is represented in semantic memory, and it is interconnected with its superordinate category animal, as well as with respective feature information of an elephant, such as its color (e.g., Leech, 1987). Consequently, processing the word elephant might result in an automatic and parallel activation of its superordinate category animal and its feature gray (Malpass \& Meyer, 2010; Palef, 1977). Thus, it is conceivable that RT to the word elephant would be shorter if both animal and gray were defined as targets than if either only animal or only gray was defined as a target. Note that the semantic targets animal and gray are not perceivable features of the proximal stimulus elephant and, thus, differ crucially from targets employed in previous studies (e.g., Mordkoff \& Danek, 2011).

Although there is one previous study (Mullin \& Egeth, 1989) that examined such semantic redundancy gain, to our knowledge, nobody has done so without manipulating the number of proximal targets. Specifically, Mullin and Egeth presented a single word on single-target trials, whereas they presented two words on redundant-target trials. Participants were asked to respond if at least one word belonged to a certain category (e.g., animal). Since RT did not differ between single- and redundant-target trials, these authors concluded that semantic categorization of words suffers from capacity limitation, resulting in no benefit for semantic redundancy. However, it is unclear whether semantic redundancy gain can be observed when only one proximal stimulus is presented on each trial that matches either a single semantic target or redundant semantic targets. Here, singleand redundant-target trials require the same amount of processing capacity at the input level.

The aim of the present experiment was to investigate semantic redundancy gain with invariant number of proximal stimuli. To this end, we adapted the go/no-go design of Mordkoff and Yantis (1991, 1993; see also Mordkoff \&
Danek, 2011), in which redundancy gains are usually observed for proximal stimuli. For each participant, a specific superordinate category (e.g., animal) was defined as one target, and a specific color (e.g., gray) was defined as another target. On each trial, a single written word was presented, and participants were asked to respond if the meaning of this word matched the target feature superordinate category (e.g., a beaver is an animal), the target feature color (e.g., a stone is typically gray), or both target features (e.g., an elephant is an animal and typically gray) but to withhold their response if the word matched neither of the two target features. Thus, in order to respond properly, participants had to retrieve the relevant target information from semantic memory, since the response decision could not be based on the mere perceptual information provided by the proximal stimulus (i.e., the word presented).

The observation of a semantic redundancy gain would indicate common cognitive operations in processing redundancy for both proximal stimuli and mental representations. In addition, a subsequent analysis of RT distributions might help to circumscribe the mechanisms of information retrieval from semantic memory. If semantic information about superordinate category and color are assessed in parallel and independently, RT distributions should be consistent with the race model inequality (Miller, 1982). By contrast, a violation of this inequality would rule out models that assume a mere race between redundant semantic targets. Finally, given the results of Mullin and Egeth (1989), it is also conceivable that semantic redundancy does not facilitate responses. Such a result, however, would indicate that cognitive operations in processing redundancy differ between proximal stimuli and mental representations.

\section{Method}

\section{Participants}

Twenty-four students at the University of Tübingen (20 female and 4 male; mean age, 20.2 years; 20 right-handed) participated in a single experimental session. They received either partial fulfillment of a course requirement or payment $(€ 7.00)$ for their participation.

\section{Stimuli}

The stimulus set consisted of 60 written German words (syllable range, 1-4; letter range, 3-12). The meaning of each word matched one specific superordinate category (animal, food, or object) and one specific color (brown, gray, or white) that were relevant for the response. For example, the word Elefant (German for elephant) matched the superordinate category animal and the color gray. Overall, there were six 
category-color combinations ${ }^{1}$ : animal-brown, animal-gray, food-brown, food-white, object-gray, and object-white. The 60 words were selected from a list of 90 words (retrieved from the CELEX databank; Baayen, Piepenbrock, \& van Rijn, 1993) on the basis of a pilot study.

\section{Apparatus}

Stimulus presentation and data collection were controlled by the Experimental Run Time System (Berisoft, Frankfurt am Main, Germany) running on a DOS computer. Responses were collected with a bounce-free response key. All stimuli were presented in yellow against a black background of a standard computer screen. A centrally presented frame $\left(6.5^{\circ}\right.$ $\times 4.0^{\circ}$ of visual angle) served as both a warning signal and a fixation frame.

\section{Procedure}

For each participant, a specific superordinate category and a specific color were defined as target features. For example, if animal and gray were defined as targets, the participants were asked to respond to any word that matched the category animal (all words from the combination animalbrown; single-target condition), the color gray (all words from the combination object-gray; single-target condition), or both (all words from the combination animal-gray; redundant-target condition) with a speeded keypress with the index finger of their dominant hand (go trials). However, they were asked to withhold their response when a word was presented that matched neither animal nor gray (all words from the combinations food-brown, food-white, and objectwhite; no-go trials). The assignment of category-color combination to single-target, redundant-target, and no-go conditions was counterbalanced across participants. ${ }^{2}$ Table 1 shows the resulting six different experimental versions.

The experiment consisted of one practice block and nine experimental blocks, with 60 trials in each. Each word was presented once per block, and the order of words was pseudorandomized within each block. Overall, there were 540 experimental trials ( 270 go trials and 270 no-go trials). On 90 go trials each, the word's meaning matched the target category, the target color, or both. On no-go trials, the word's meaning matched neither the target category nor

\footnotetext{
${ }^{1}$ To control for stimulus and response contingency effects, it is only possible to use six out of the nine possible category-color combinations (see Mordkoff \& Danek, 2011; Mordkoff \& Yantis, 1991, 1993).

2 The present design prevented biases in favor of redundancy gain, since each category-color combination constitutes a single-target condition twice as often as it constitutes a redundant-target condition. Hence, if responses to members of a specific category-color combination are especially fast (e.g., due to word frequency effects), this will diminish the redundancy gain twice as often as it facilitates the redundancy gain.
}

the target color. Table 2 shows the proportion of trials per condition, which follows the recommendations of Mordkoff and Yantis (1991) for assessing the redundancy gain in a go/ no-go task in order to avoid interstimulus contingencies and nontarget response contingencies.

Each trial started with the presentation of the fixation frame. After 1,000 ms, a word appeared within the fixation frame until a response was registered or until $2,000 \mathrm{~ms}$ had elapsed. If participants responded too quickly (anticipations, $\mathrm{RT}<110 \mathrm{~ms}$ ) or too slowly (misses, RT $>2,000$ ) on go trials, respective written feedback was provided for $1,500 \mathrm{~ms}$. In the case of an erroneous response on no-go trials (false alarm), feedback was presented for 3,500 ms (e.g., "Bitte reagiere nur auf GRAU und/oder TIER!"; German for "Please respond only to GRAY and/or ANIMAL!"). The next trial started after an intertrial interval of 900-ms duration, during which no stimulus was presented on the screen. After each block, participants were given the opportunity to take a short rest before they initiated the next block with a keypress.

\section{Results}

Inspection of error data revealed that there were no anticipations $(0.0 \%)$, low miss rates $(0.4 \%$ of all go trials), and low false alarm rates ( $2.0 \%$ of all no-go trials). Both the low miss and low false alarm rates indicate that participants could easily decide whether the words' meanings matched the respective target category and/or target color. Furthermore, the observation of more false alarms than misses is typical in go/no-go tasks and probably reflects the fact that participants occasionally fail to withhold a prepotent response. Figure 1 shows the resulting mean RTs for the two single-target conditions and the redundant-target condition. A one-way repeated measures ANOVA (with Greenhouse-Geisser corrected $p$-values) on RT with the factor of condition (single-category condition vs. single-color condition vs. redundant-target condition) revealed a significant main effect of condition, $F(2,46)=$ 14.58, $M S E=874.47, p<.001$. Planned contrasts (Fishers LSD; critical value: $17.2 \mathrm{~ms}, p<.05$ ) revealed that RT was shorter for the redundant-target condition $(535 \mathrm{~ms})$ than for both the single-category condition $(573 \mathrm{~ms})$ and the singlecolor condition $(577 \mathrm{~ms})$, whereas RT did not differ for the two single-target conditions.

In order to assess the redundancy gain, mean RT for the faster of each participant's two single-target conditions was compared against the mean RT in the redundant-target condition. This comparison is a standard procedure for preventing an artificially increased redundancy gain due to averaging across some participants who responded faster in the singlecategory condition (13 out of 24 participants in the present study) and other participants who responded faster in the 
Table 1 Experimental versions and corresponding assignment of category-color combinations to redundant-target, single-target, and no-go conditions

\begin{tabular}{|c|c|c|c|c|}
\hline Experimental version & Redundant targets & Single-target category & Single-target color & No-go \\
\hline 1 & $\mathrm{AB}$ & AG & FB & FW, OG, OW \\
\hline 2 & $\mathrm{AG}$ & $\mathrm{AB}$ & OG & FB, FW, OW \\
\hline 3 & FB & FW & $\mathrm{AB}$ & AG, OG, OW \\
\hline 4 & FW & FB & OW & $\mathrm{AB}, \mathrm{AG}, \mathrm{OG}$ \\
\hline 5 & OG & OW & AG & $\mathrm{FB}, \mathrm{FW}, \mathrm{AB}$ \\
\hline 6 & OW & OG & FW & $\mathrm{FB}, \mathrm{AB}, \mathrm{AG}$ \\
\hline
\end{tabular}

For each version, the corresponding category-color combinations in the redundant-target condition, the single-target category condition, the singletarget color condition, and the no-go conditions are listed. $\mathrm{A}=$ animal; $\mathrm{F}=$ food; $\mathrm{O}=$ object; $\mathrm{B}=$ brown; $\mathrm{G}=$ gray; $\mathrm{W}=$ white

single-color condition (cf. Biederman \& Checkosky, 1970). A paired $t$-test revealed that mean RT was significantly shorter for the redundant-target $(535 \mathrm{~ms})$ than for the faster singletarget (556 ms) condition, $t(23)=3.39, p<.005$, reflecting a redundancy gain for semantic target features.

Next, we tested whether practice had an effect on the size of the redundancy gain. Since each word was presented 10 times within the entire experiment, one could assume that participants have learned the target words by heart after a few blocks of trials. If true, participants could have based their responses merely on low-level features of the target words, such as the shape of letter strings. In this case, no redundancy gain should result in blocks of trials at the end of the experiment. In order to investigate this possibility, we ran another ANOVA with the factor of condition (faster single vs. redundant) and the additional factor of practice (blocks $1-4$ vs. blocks $6-9$ ). ${ }^{3}$ This analysis revealed that participants responded significantly faster in the last four blocks $(519 \mathrm{~ms})$ than in the first four blocks $(571 \mathrm{~ms})$ of the experiment, $F(1,23)=53.32, M S E=1,198.8, p<.001$. As in the previous analysis, the effect of condition was significant, $F(1,23)=11.26, M S E=953.3, p<.005$, reflecting a redundancy gain of $21 \mathrm{~ms}$. The interaction of condition and practice was also significant, $F(1,23)=6.73, M S E=$ 236.51, $p<.05$. Redundancy gain was larger in the first four blocks $(30 \mathrm{~ms})$ than in the last four blocks $(13 \mathrm{~ms})$ of the experiment. Post hoc $t$-tests revealed, however, that the size of the redundancy gain was significantly different from zero for both the first four blocks, $t(23)=3.60, p<.005$, and the last four blocks, $t(23)=2.27, p<.05$, of the experiment. Thus, we argue that participants responded on the basis of semantic rather than on the basis of mere perceptual information throughout the entire experiment.

To assess potential redundancy effects at the distributional level, Vincentized RT distributions were computed for the single and redundant conditions (cf. Ulrich, Miller, \&

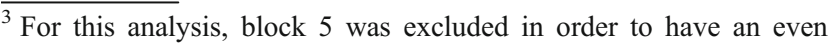
number of blocks per factor level.
}

Schröter, 2007), and these are shown in Fig. 2. Also shown in the figure is the sum of the Vincentized single-target cumulative density functions used to test the race model inequality. Paired $t$-tests conducted at each of 20 percentile points (percentiles 2.5, 7.5, up to 97.5) revealed that the present data did not violate this inequality (all $p \mathrm{~s}>.05$ ). ${ }^{4}$ This finding was also confirmed by a horizontal noninferiority test (criterion $\varepsilon=1 \mathrm{~ms}$, all $p \mathrm{~s}<.05$ ) in which the race model prediction is used as the alternative hypothesis (for further details, see Gondan, Riehl, \& Blurton, 2012). Both tests yielded identical patterns of statistical reliability when only the RT data for the first four blocks of trials were included. Thus, the data are consistent with parallel and independent processing of superordinate category and color.

\section{Discussion}

Until now, redundancy gains on RT have been observed only for responses to proximal stimuli (e.g., Fischer \& Miller, 2008). The present study investigated whether such gains also emerge when participants process semantic information. In a previous study, Mullin and Egeth (1989) did not find a semantic redundancy gain. As was mentioned in the Introduction, this null result cannot be interpreted unequivocally as evidence that redundant proximal stimuli and mental representations are processed by different cognitive operations, because capacity requirements were especially high for redundant-target trials in this previous study. In order to avoid differences in capacity requirements at the input level, the present study used a go/no-go design in which a single word was presented on each trial. Participants were asked to respond to the word if its meaning matched a specific target category, a specific target color, or both. Importantly, the target features were not presented

\footnotetext{
${ }^{4}$ Shepherdson and Miller (2012) have recently reported data for a different semantic categorization task that are in line with the present findings. These authors did not observe a violation of the race inequality either.
} 
Table 2 Proportion of trials per condition

\begin{tabular}{|c|c|c|c|}
\hline \multirow[t]{2}{*}{ Color feature } & \multicolumn{3}{|c|}{ Category feature } \\
\hline & $\begin{array}{l}\text { Target } \\
\text { (e.g., animal) }\end{array}$ & $\begin{array}{l}\text { Nontarget } 1 \\
\text { (e.g., object) }\end{array}$ & $\begin{array}{l}\text { Nontarget } 2 \\
\text { (e.g., food) }\end{array}$ \\
\hline Target (e.g., gray) & .167 & .167 & 0 \\
\hline $\begin{array}{l}\text { Nontarget } 1 \\
\text { (e.g., brown) }\end{array}$ & .167 & 0 & .167 \\
\hline $\begin{array}{l}\text { Nontarget } 2 \\
\text { (e.g., white) }\end{array}$ & 0 & .167 & .167 \\
\hline
\end{tabular}

in the extrapersonal world; that is, they were not perceivable features of the proximal stimulus but, instead, had to be retrieved from semantic memory. In contrast to Mullin and Egeth, a reliable semantic redundancy gain was obtained, suggesting that the processing of mental representations can benefit from redundancy just like the processing of proximal stimuli.

As was discussed in the Introduction, this semantic redundancy gain could stem from a parallel and independent retrieval of the two target features from semantic memory. Specifically, following the encoding of the presented word, a subsequent race between an achromatic category analyzer, on the one hand, and a chromatic analyzer, on the other hand, would result in a redundancy gain that is limited to mere statistical facilitation. An alternative view involves the notion of preactivation due to semantic priming (e.g., Meyer \& Schvaneveldt, 1971). Accordingly, all those concepts stored in semantic memory that match one or both of the two target features (i.e., everything that is an animal and/or everything that is gray) might have been preactivated. This should shorten RTs in both single- and redundant-target

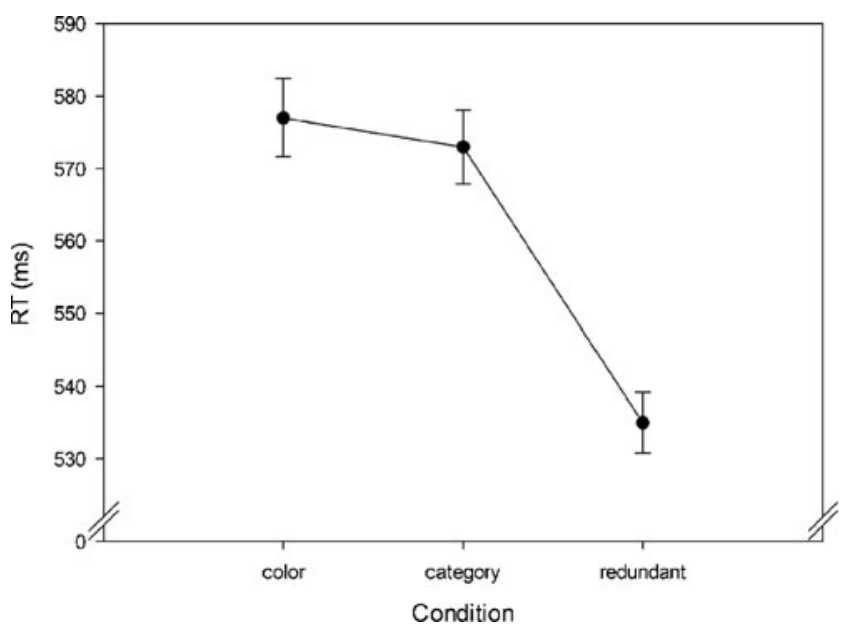

Fig. 1 Mean response time (RT) as a function of condition (singlecategory condition, single-color condition, redundant-target condition). Error bars show the within-subjects standard errors of the means, using an algorithm suggested by Cousineau (2005) conditions, because the response criterion is reached earlier for preactivated concepts. Crucially, this reduction in RT should be especially prominent in the redundant-target condition, because the respective concepts would benefit twice from preactivation. The resulting redundancy gain predicted by this model might even exceed mere statistical facilitation. Finally, there could be a combination of preactivation and subsequent race of detection processes for color and superordinate category. These combined mechanisms would most likely result in a redundancy gain that violates the race model inequality.

The analysis of RT distribution revealed that the present data clearly obeyed the race model inequality. This result is most consistent with the notion of parallel and independent retrieval of information from semantic memory. In principle, a model of exclusive preactivation with a moderate amount of semantic priming might also account for the data obtained. In contrast, the results seem to be less consistent with a model that assumes combined mechanisms of preactivation and subsequent race of detection processes.

Future work should examine whether semantic redundancy gain is the result of preactivation processes or of a race between semantic targets. For instance, to our knowledge, it has yet to be shown whether redundant preactivation of semantic concepts is possible at all. In order to test this possibility, one could conduct a lexical decision task and present two primes at the beginning of each trial (e.g., animal and gray). Following the primes, a letter string (e.g., milk, stone, elephant, or a nonword such as pfum) could be presented, and participants would decide whether the letter string is a word. In this task, a typical priming effect should occur - that is, faster responses to words that match the category prime, the color prime, or both. Crucially, if redundant preactivation is possible, responses should

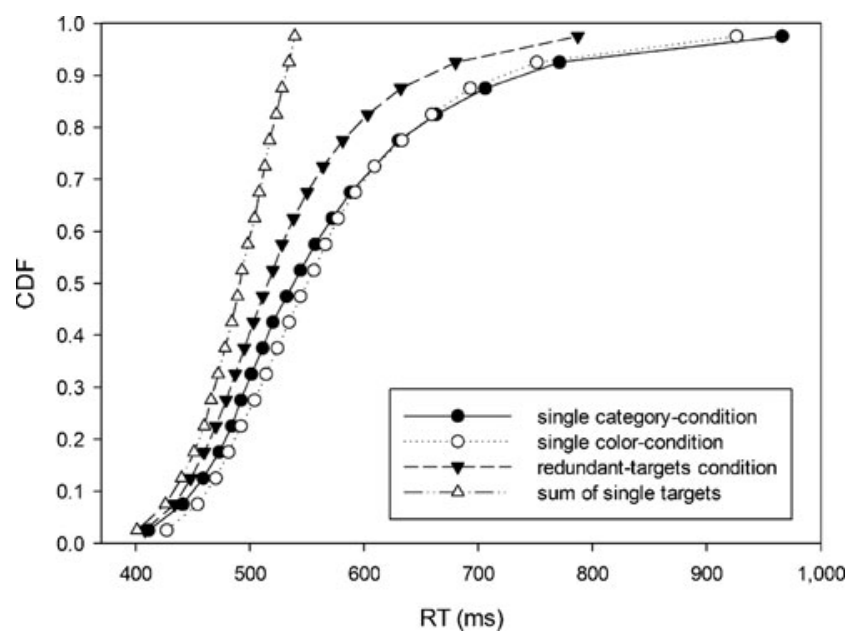

Fig. 2 Estimated cumulative density function (CDF) for reaction time (RT) as a function of stimulus condition 
be especially fast for target words that match both the category and the color primes.

Although we have favored a parallel race model to account for the present results, it should be noted that the data are also consistent with a random-order serial-decision model (see van Zandt \& Townsend, 1993). According to this model, participants perform a serial self-terminating search and check for the color target and the category target in a random order. On redundant-target trials, a target is always detected in the first step of the search. In contrast, on singletarget trials, a target is detected only on half of all trials in the first step. On the other half of trials, two search steps are required for detection. Consequently, on average, RT is longer on single-target trials than on redundant-target trials. It is difficult, if not impossible, however, to empirically discriminate parallel and serial retrieval models on the basis of RT (e.g., Townsend \& Ashby, 1983; Vorberg, 1977). In several important metatheoretical contributions, Townsend and colleagues have shown that parallel-processing models can mimic the prediction of serial models (Townsend, 1971; Townsend \& Ashby, 1983; see also McGill, 1963; Vorberg \& Ulrich, 1987) and even a violation of the RMI can be explained by assuming a specific serial-processing architecture (Townsend \& Nozawa, 1997).

To sum up, the present study provides the first evidence of redundancy gain for semantic targets with a single proximal stimulus presented in each condition. This result shows that redundancy gain is also inherent in cognitive mechanisms that process stored information in memory, rather than external information that stimulates our senses.

Acknowledgements This study was supported by the Deutsche Forschungsgemeinschaft (SCHR 1180/2-2). We thank Barbara Kaup, Hartmut Leuthold, and Jeff Miller for helpful comments and suggestions on an earlier version of the manuscript.

\section{References}

Baayen, R. H., Piepenbrock, R., \& van Rijn, H. (1993). The CELEX lexical database. Philadelphia: Linguistic Data Consortium, University of Pennsylvania.

Biederman, I., \& Checkosky, S. F. (1970). Processing redundant information. Journal of Experimental Psychology, 83, 486-490. doi: $10.1037 / \mathrm{h} 0028841$

Cousineau, D. (2005). Confidence intervals in within-subject designs: A simpler solution to Loftus and Masson's method. Tutorials in Quantitative Methods for Psychology, 1, 42-45.

Diederich, A., \& Colonius, H. (2004). Bimodal and trimodal multisensory enhancement of reaction time: Effects of stimulus onset and intensity. Perception \& Psychophysics, 66, 1388-1404. doi:10.3758/BF03195006

Fiedler, A., O’Sullivan, J., Schröter, H., Miller, J., \& Ulrich, R. (2011). Illusory double flashes can speed up responses like physical ones: Evidence from the sound-induced flash illusion. Experimental Brain Research, 214, 113-119. doi:10.1007/s00221-011-2811-z
Fischer, R., \& Miller, J. O. (2008). Differential redundancy gain in onset detection versus offset detection. Perception \& Psychophysics, 70, 431-436. doi:10.3758/PP.70.3.431

Gilford, R. M., \& Juola, J. F. (1976). Familiarity effects on memory search and visual search. Bulletin of the Psychonomic Society, 7, $142-144$.

Gondan, M., Riehl, V., \& Blurton, S. P. (2012). Showing that the race model inequality is not violated. Behavioral Research Methods, 44, 248-255. doi:10.3758/s13428-011-0147-z

Leech, G. (1987). Semantics. The study of meaning. Harmondsworth: Penguin Books.

Malpass, D., \& Meyer, A. S. (2010). The time course of name retrieval during multiple-object naming: Evidence from extrafoveal-onfoveal effects. Journal of Experimental Psychology: Learning, Memory, and Cognition, 36, 523-537. doi:10.1037/a0018522

McGill, W. J. (1963). Stochastic latency mechanisms. In R. D. Luce, R. B. Bush, \& E. Galanter (Eds.), Handbook of mathematical psychology (Vol. 1). New York: Wiley.

Meyer, D. E., \& Schvaneveldt, R. W. (1971). Facilitation in recognizing pairs of words: Evidence of a dependence between retrieval operations. Journal of Experimental Psychology, 90, 227-234. doi: $10.1037 / \mathrm{h} 0031564$

Miller, J. (1982). Divided attention: Evidence for coactivation with redundant signals. Cognitive Psychology, 14, 247-279. doi:10.1016/ 0010-0285(82)90010-X

Mordkoff, J. T., \& Danek, R. H. (2011). Dividing attention between color and shape revisited: Redundant targets coactivate only when parts of the same perceptual object. Attention, Perception, \& Psychophysics, 73, 103-112. doi:10.3758/s13414-010-0025-2

Mordkoff, J. T., \& Yantis, S. (1991). An interactive race model of divided attention. Journal of Experimental Psychology. Human Perception and Performance, 17, 520-538.

Mordkoff, J. T., \& Yantis, S. (1993). Dividing attention between color and shape: Evidence of coactivation. Perception \& Psychophysics, 53, 357-366. doi:10.3758/BF03206778

Mullin, P. A., \& Egeth, H. E. (1989). Capacity limitations in visual word processing. Journal of Experimental Psychology. Human Perception and Performance, 15, 111-123. doi:10.1037/0096-1523.15.1.111

Palef, S. R. (1977). Searching memory for physical and semantic information. Canadian Journal of Psychology/Revue canadienne de psychologie, 31, 131-138.

Raab, D. H. (1962). Statistical facilitation of simple reaction times. Transactions of the NewYork Academy of Sciences, 24, 574-590.

Schröter, H., Ulrich, R., \& Miller, J. (2007). Effects of redundant auditory stimuli on reaction time. Psychonomic Bulletin and Review, 14, 39-44. doi:10.3758/BF03194025

Schröter, H., Frei, L. S., Ulrich, R., \& Miller, J. O. (2009). The auditory redundant signals effect: An influence of number of stimuli or number of percepts? Attention, Perception, \& Psychophysics, 71, 1375-1384. doi:10.3758/APP.71.6.1375

Schröter, H., Fiedler, A., Miller, J., \& Ulrich, R. (2011). Fusion prevents the redundant signals effect: Evidence from stereoscopically presented stimuli. Journal of Experimental Psychology. Human Perception and Performance, 37, 1361-1368. doi:10.1037/a0024280

Shams, L., Kamitani, Y., \& Shimojo, S. (2002). Visual illusion induced by sound. Cognitive Brain Research, 14, 147-152. doi:10.1016/ S0926-6410(02)00069-1

Shepherdson, P. V., \& Miller, J. O. (2012). Redundancy gain in semantic categorization. Abstracts of the 53rd annual meeting of the Psychonomic Society, 17, 188.

Töllner, T., Zehetleitner, M., Krummenacher, J., \& Müller, H. J. (2011). Perceptual basis of redundancy gains in visual pop-out search. Journal of Cognitive Neuroscience, 23, 137-150. doi:10.1162/jocn.2010.21422

Townsend, J. T. (1971). A note on the identifiability of parallel and serial processes. Perception \& Psychophysics, 10, 161-163. 
Townsend, J. T., \& Ashby, F. G. (1983). The stochastic modeling of elementary psychological processes. New York: Cambridge Univ. Press.

Townsend, J. T., \& Nozawa, G. (1997). Serial exhaustive models can violate the race model inequality: Implications for architecture and capacity. Psychological Review, 104, 595-602.

Tulving, E. (1983). Elements of episodic memory. Oxford: Oxford University Press.

Ulrich, R., Miller, J., \& Schröter, H. (2007). Testing the race model inequality: An algorithm and computer programs. Behavior Research Methods, 39, 291-302. doi:10.3758/BF03193160
Van Zandt, T., \& Townsend, J. T. (1993). Self-terminating versus exhaustive processes in rapid visual and memory search: An evaluative review. Perception \& Psychophysics, 53, 563-580. doi:10.3758/BF03205204

Vorberg, D. (August 1977). On the equivalence of parallel and serial models of information processing. Paper presented at the Tenth Mathematical Psychology Meetings, Los Angeles.

Vorberg, D., \& Ulrich, R. (1987). Random search with unequal search rates: Serial and parallel generalizations of McGill's model. Journal of Mathematical Psychology, 31, 1-23. doi:10.1016/00222496(87)90033-2 